\title{
Anti-message Logging based Check Pointing Algorithm for Mobile Distributed Systems
}

\author{
Monika Nagpal, PhD. \\ CMJ university
}

\begin{abstract}
Checkpointing is one of the commonly used techniques to provide fault tolerance in distributed systems so that the system can operate even if one or more components have failed. However, mobile computing systems are constrained by low bandwidth, mobility, lack of stable storage, frequent disconnections and limited battery life. Hence checkpointing protocols which have fewer checkpoints are preferred in mobile environment. In this paper, we propose a minimum-process coordinated Checkpointing algorithm for checkpointing deterministic distributed applications on mobile systems. We eliminate useless checkpoints as well as blocking of processes during checkpoints at the cost of logging anti-messages of very few messages during Checkpointing. We also try to minimize the loss of checkpointing effort.
\end{abstract}

\section{INTRODUCTION}

In deterministic systems, if two processes start in the same state, and both receive the identical sequence of inputs, they will produce the identical sequence outputs and will finish in the same state. The state of a process is thus completely determined by its starting state and by sequence of messages it has received [10, 11, 12]. Johnson and Zwaenepoel [11] proposed sender based message logging for deterministic systems, where each message is logged in volatile memory on the machine from which the message is sent. The massage log is then asynchronously written to stable storage, without delaying the computation, as part of the sender's periodic checkpoint. Johnson and Zwaenepoel [12] used optimistic message logging and checkpointing to determine the maximum recoverable state, where every received message is logged. David R. Jefferson [10] introduced the concept of antimessage. Anti-message is exactly like an original message in format and content except in one field, its sign. Two messages that are identical except for opposite signs are called anti-messages of one another. All messages sent explicitly by user programs have a positive (+) sign; and their anti-messages have a negative sign (-). Whenever a message and its anti-message occur in the same queue, they immediately annihilate one another. Thus the result of enqueueing a message may be to shorten the queue by one message rather than lengthen it by one. We depict the antimessage of $\mathrm{m}_{\text {by }} \mathrm{m}^{-1}$.

In this paper, we propose a minimum-process coordinated Checkpointing algorithm for Checkpointing deterministic distributed applications on mobile systems. We eliminate useless checkpoints as well as blocking of processes during checkpoints at the cost of logging anti-messages of very few messages during Checkpointing. We also try to minimize the loss of checkpointing effort. Frequent aborts of checkpointing procedure may happen in mobile systems due to exhausted battery, non-voluntary disconnections of MHs, or poor wireless connectivity. Therefore, we propose

\author{
Praveen Kumar, PhD. \\ MIET \\ Meerut (India)
}

that in the first phase, all concerned MHs will take ad hoc checkpoint only. In case of an $\mathrm{MH}$, ad hoc checkpoint is stored on the memory of MH only. In this case, if some process fails to take checkpoint in the first phase, then MHs need to abort their ad hoc checkpoints only. In this way, we try to minimize the loss of checkpointing effort when any process fails to take its checkpoint in coordination with others.

\subsection{Problems in the Existing Algorithms}

Singh and Cabillic [13] proposed a checkpointing algorithm for mobile computing environments on the basis of anti-message logging. This algorithm may lead to inconsistencies as follows. In Figure 1.1, at time $\mathrm{t}_{0}, \mathrm{P}_{1}$ initiates checkpointing. Since, it has received $\mathrm{m}_{1}$ and $\mathrm{m}_{2}$ from $\mathrm{P}_{0}$ and $\mathrm{P}_{2}$, respectively, since its last permanent checkpoint $\mathrm{C}_{11}$; therefore, $\mathrm{P}_{1}$ sends checkpoint request to $\mathrm{P}_{0}$ and $\mathrm{P}_{2}$. When $\mathrm{P}_{0}$ receives the checkpoint request from $\mathrm{P}_{1}$, it finds that it has not sent any message to $\mathrm{P}_{1}$ since its last permanent checkpoint $\mathrm{C}_{02}$. Therefore, $\mathrm{P}_{0}$ discards the checkpoint request. $P_{2}$ receives $m_{3}$ without logging its antimessage. When $\mathrm{P}_{2}$ receives the checkpoint request from $\mathrm{P}_{1}$, it takes its tentative checkpoint $\mathrm{C}_{23}$, because, it has sent $\mathrm{m}_{2}$ to $\mathrm{P}_{1}$ since its last permanent checkpoint $\mathrm{C}_{22}$. After taking its tentative checkpoint, $\mathrm{P}_{2}$ finds that it has received $\mathrm{m}_{3}$ from $\mathrm{P}_{0}$ and $\mathrm{P}_{0}$ has already been sent the checkpoint request; therefore, $\mathrm{P}_{2}$ does not send the checkpoint request to $P_{0}$. In this way, $\left\{C_{02}, C_{12}, C_{23}\right\}$ constitute a recovery line, where $\mathrm{m}_{3}$ is an orphan message without its antimessage being logged at $\mathrm{P}_{2}$. Hence, the algorithm [85] may lead to inconsistencies.

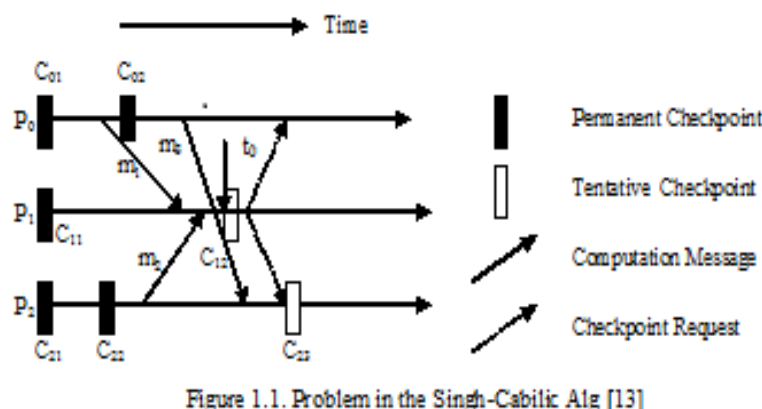

\section{THE PROPOSED CHECKPOINTING ALGORITHM}

\subsection{System Model}

There are $n$ spatially separated sequential processes denoted by $P_{0}, P_{l}, . ., P_{n-1}$, running on MHs or MSSs, constituting a mobile distributed computing system. Each MH/MSS has one process running on it. The processes do not share memory or clock. Message passing is the only way for processes to communicate with each other. Each process progresses at its own speed and messages are 
exchanged through reliable channels, whose transmission delays are finite but arbitrary. We also assume that the processes are deterministic as in [11], [12], [13].

\subsection{Basic Idea}

During the checkpointing procedure, a process $\mathrm{P}_{\mathrm{i}}$ may receive $m$ from $P_{j}$ such that $P_{j}$ has taken its tentative checkpoint for the current initiation whereas $\mathrm{P}_{\mathrm{i}}$ has not taken. If $P_{i}$ processes $m$ and it receives checkpoint request later on and takes its checkpoint, then $\mathrm{m}$ will become orphan in the recorded global state. In order to avoid such orphan messages, Cao and Singhal [2] proposed that $\mathrm{P}_{\mathrm{P}}$ should take a forced checkpoint before processing $\mathrm{m}$. If $\mathrm{P}_{\mathrm{i}}$ receives a checkpoint request after processing $\mathrm{m}$, then the forced checkpoint already taken is converted into tentative one. In this way, $\mathrm{m}$ will not become orphan. P Kumar [9] proposed that such messages should be buffered at the receiver end. The receiver should process such messages only after taking its checkpoint or after getting conformed that it is not going to take its checkpoint in the current initiation. Koo-Toueg [4] proposed that $P_{j}$ should not send any computation message to any process after taking its checkpoint for the current initiation. $P_{j}$ starts sending messages only after getting conformed that all concerned processes have taken their checkpoint for the current initiation. We propose that the anti-messages of only those messages, which can become orphan, should be recorded at the receiver end. In deterministic systems, orphan messages are received as duplicate messages on recovery. A duplicate message is annihilated by its antimessage at the receiver end before processing. Hence, in deterministic distributed systems, an orphan message in global checkpoint does not create any inconsistency during recovery if its anti-message is logged at the receiver end. By doing so, we avoid the blocking of processes as well as the useless checkpoints in minimum-process checkpointing. It should be noted that in minimum-process coordinated checkpointing, some useless checkpoints are taken $[2,8,14]$ or blocking of processes takes place $[4,6$, 9]. The overheads of logging a few anti-messages may be negligible as compared to taking some useless checkpoints or blocking the processes during checkpointing.

The initiator MSS computes int_vect [subset of the minimum set] on the basis of dependencies maintained locally; and sends the checkpoint request along with the int_vect[] to the relevant MSSs. On receiving checkpoint request, an MSS asks concerned processes to checkpoint and computes new processes for the minimum set. By using this technique, we have tried to optimize the number of messages between MSSs. When the initiator MSS commits the checkpointing process, it sends the commit request along with the exact minimum set to all MSSs and every MSS maintains up-to-date comm_csn_vect[]. comm_csn_vect[] is described in Section 4.5. This enables us to maintain exact dependencies among processes. In our protocol, $\mathrm{cv}_{\mathrm{i}}[\mathrm{j}]=1$ only if $\mathrm{P}_{\mathrm{i}}$ is directly dependent upon $\mathrm{P}_{\mathrm{j}}$ in the current $\mathrm{CI}$. Therefore, useless checkpoint requests, as occur in [2], are not sent in our algorithm.

When $P_{i}$ sends c_req to $P_{j}$, it also piggybacks $\operatorname{csn}_{i}[j]$ [2]. When $P_{j}$ receives c_req, it decides, on the basis of

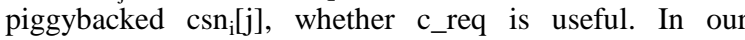
protocol, no useless c_req is sent, therefore, $\operatorname{csn}_{i}[j]$ is not piggybacked onto c_req.

In algorithm [2], when a process, say $\mathrm{P}_{\mathrm{j}}$, takes its tentative checkpoint, it also finds the processes $P_{k}$ such that $P_{j}$ has received $m$ from $P_{k}$ in the current CI. On the basis of $M R$, received with the checkpoint request, $P_{j}$ decides the following: (i) whether any process has already sent the checkpoint request to $\mathrm{P}_{\mathrm{k}}$ (ii) whether the earlier checkpoint request to $\mathrm{P}_{\mathrm{k}}$ is useless. In our protocol, no useless checkpoint request is sent, therefore, data structures MR[] is not piggybacked onto checkpoint requests. The decision (i) is taken on the basis of tint_vect, maintained at every MSS. tint_vect maintains the local knowledge about the minimum set. In our case, instead of MR[], tint_vect is piggybacked onto checkpoint requests. The size of the tint_vect is negligibly small as compared to MR[].

In coordinated checkpointing, if a single process fails to take its checkpoint; all the checkpointing effort goes waste, because, each process has to abort its tentative checkpoint. Furthermore, in order to take the tentative checkpoint, an MH needs to transfer large checkpoint data to its local MSS over wireless channels. Hence, the loss of checkpointing effort may be exceedingly high due to frequent aborts of checkpointing algorithms especially in mobile systems. In mobile distributed systems, there remain certain issues like: abrupt disconnection, exhausted battery power, or failure in wireless bandwidth. So there remains a good probability that some $\mathrm{MH}$ may fail to take its checkpoint in coordination with others. Therefore, we propose that in the first phase, all processes in the minimum set, take ad hoc checkpoint only. Ad hoc checkpoint is stored on the memory of MH only. If some process fails to take its checkpoint in the first phase, then other MHs need to abort their ad hoc checkpoints only. The effort of taking an ad hoc checkpoint is negligible as compared to the tentative one. In this second phase, a process converts its ad hoc checkpoint into tentative one. By using this scheme, we try to minimize the loss of checkpointing effort in case of abort of checkpointing algorithm in the first phase.

\subsection{Data Structures}

Here, we describe the data structures used in the checkpointing protocol. A process that initiates checkpointing, is called initiator process and its local MSS is called initiator MSS. If the initiator process is on an MSS, then the MSS is the initiator MSS. Data structures are initialized on the completion of a checkpointing process if not mentioned explicitly. We use the term potential checkpoint request to an MSS, if at least one process takes a checkpoint in its cell to this request.

i) Each process $P_{i}$ maintains the following data structures, which are preferably stored on local MSS :

p_cn $\mathbf{n}_{\mathbf{i}}$ an integer; it is a process csn; on tentative

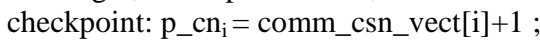
on commit or abort: after updating comm_csn_vect[]

p_cn $=$ icomm_csn_vect $[\mathrm{i}]$

; comm_csn_vect[] is described later described later ;

$\mathbf{c v}_{\mathrm{i}}\left[\mathrm{]}: \quad \quad \mathrm{cv}_{\mathrm{i}}[\mathrm{j}]=1\right.$ implies $\mathrm{P}_{\mathrm{i}}$ is causally dependent upon $P_{j} \cdot c v_{i}[j]$ is set to ' 1 ' only if $P_{i}$ processes $m$ received from $P_{j}$ such that m.p_cn $\geq$ comm_csn_vect[j]; m.p_cn is the $p_{-} c n$ at $P_{j}$ at the time of sending $m$ and omm._csn_vect[j] is $P_{j}$ 's recent permanent checkpoint's omm._csn_vect; initially for $\mathrm{P}_{\mathrm{i}}, \forall \mathrm{k}, \mathrm{cv}_{\mathrm{i}}[\mathrm{k}]=0$ and $\mathrm{cv}_{\mathrm{i}}[\mathrm{i}]=1$; for $\mathrm{MH}_{\mathrm{i}}$ it is kept at local MSS; maintenance of $\mathrm{cv}[]$ is described in Section 
4.5.2;

tentative $_{\mathbf{i}}$ a flag; set to ' 1 ' on tentative checkpoint;

adhoc $_{\mathbf{i}}$ a flag; set to ' 1 ' on ad hoc checkpoint;

(ii) Initiator MSS (any MSS can be initiator MSS) maintains the following Data structures:

int_vect[] a bit vector of size $\mathrm{n}$; int_vect[k]=1 implies $\mathrm{P}_{\mathrm{k}}$ belongs to the minimum set; initially, int_vect[] (subset of the minimum set ) is computed by using $\mathrm{cv}$ vectors maintained at the initiator MSS ; on receiving response() from some MSS: int_vect=int_vect $\cup \quad n p \_i n t \_v e c t ; \quad$ after receiving responses from all relevant processes, int_vect[] contains the exact minimum set; ' $\cup$ ', is a operator for bitwise logical OR; np_int_vect is described later;

R[]: $\quad$ a bit vector of length $n ; R[i]=1$ implies $P_{i}$ has taken its ad hoc checkpoint;

timer1: a flag; initialized to ' 0 ' when the timer is set; set to ' 1 ' when maximum allowable time for collecting coordinated checkpoint expires;

T[] a bit vector of length $n ; T[i]=1$ implies $P_{i}$ has taken its tentative checkpoint;

(iii) Each MSS (including initiator MSS) maintains the following data structures:

D[]:

a bit vector of length $\mathrm{n}$; $\mathrm{D}[\mathrm{i}]=1$ implies

chkpt a flag; set to 1 when the MSS learns that some checkpointing process is going on;

ad_req a checkpoint request; when $\mathrm{MSS}_{\text {in }}$ sends ad hoc checkpoint request (ad_req) to $\mathrm{MSS}_{\mathrm{p}}$, it piggybacks the data structures: $\mathrm{P}_{\mathrm{in}}, \mathrm{MSS}_{\mathrm{in}}, \mathrm{p} \_\mathrm{cn}_{\mathrm{in}}$, MSS $_{\mathrm{p}}$, int_vect; any other MSS piggybacks tint_vect, tnp_int_vect in place of int_vect;

\subsubsection{Computation of int_vect or tnp_int_vect:}

Let $D$ be the bit dependency matrix of $n * n$, where $j^{\text {th }}$ row denote the $c v[]$ of $\mathrm{P}_{\mathrm{j}}$. For making dependency matrix at an MSS, if a process, say $\mathrm{P}_{k}$, is not in the cell of MSS, then its initial $\mathrm{cv}[]$ vector is assumed. Initial $\mathrm{cv}[]$ of $\mathrm{P}_{\mathrm{k}}$ is: $\forall \mathrm{i}$, $\operatorname{cv}[\mathrm{i}]=0 ; \operatorname{cv}[\mathrm{k}]=1$.

(a) process.

Computation of int_vect[]: Let $\mathrm{P}_{\mathrm{i}}$ be the initiator

$\mathrm{A}=\mathrm{cv}_{\mathrm{i}}[] ;$ int_vect $=\mathrm{cv}_{\mathrm{i}}[] ; \mathrm{A}=\mathrm{A} \times \mathrm{D}$;

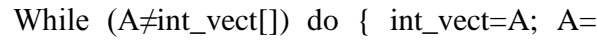

$\mathrm{A} \times \mathrm{D} ;\}$

(p) is $\quad$ Computation of tnp_int_vect: running in the cell of $\mathrm{MSS}$; it also includes the $\mathrm{A}^{\mathrm{A}}$ tint_vect; $\mathrm{B}=$ tint_vect; $\mathrm{B}=\mathrm{B} \times \mathrm{D}$; disconnected MHs supported by this MSS;

EE[]: $\quad$ a bit vector of length $n$; $E E[i]$ is set to ' 1 ' if $P_{i}$ is in its cell and it has taken its ad hoc checkpoint;

E[]: $\quad$ a bit vector of length $n$; $E[i]$ is set to ' 1 ' if ad hoc

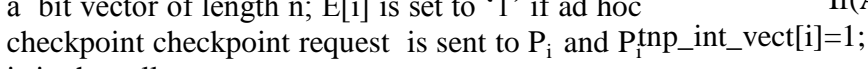
is in the cell;

F[] a bit vector of length $\mathrm{n}$; $\mathrm{F}[\mathrm{i}]$ is set to ' 1 ' if theBrief Description of the Algorithm along with an tentative checkpoint request is sent to $\mathrm{P}_{\mathrm{i}}$;

FF[] a bit vector of length $n$; FF[i] is set to ' 1 ' if $P_{i}$ is in its cell and it has taken its tentative checkpoint; We explain our checkpointing algorithm with the help of

S_bit: a flag; set to ' 1 ' when some relevant process in itsan example. In Figure 2 , at time $t_{1}, P_{2}$ initiates cell fails to take its tentative checkpoint; checkpointing process. $\mathrm{cv}_{2}[1]=1$ due to $\mathrm{m}_{1}$; and $\mathrm{cv}_{1}[4]=1$ initiator process identification

$P_{\text {in: }}$ :

MSS $_{\text {in }}$

$\mathbf{p} \__{-} \mathbf{c n}_{\text {in }}$ comm_csn_v ect[] initiator MSS identification; P_Cnof initiator process; due to $\mathrm{m}_{2}$. On the receipt of $\mathrm{m}_{0}, \mathrm{P}_{2}$ does not set $\mathrm{cv}_{2}[3]=1$, because, $\mathrm{P}_{3}$ has taken its permanent checkpoint after sending $\mathrm{m}_{0}$. We assume that $\mathrm{P}_{1}$ and $\mathrm{P}_{2}$ are in the cell of the an array of length $\mathrm{n}$ for $\mathrm{n}$ processes;same MSS, say MSS ${ }_{\mathrm{in}}$. MSS $\mathrm{in}_{\mathrm{in}}$ computes int_vect (subset of comm_csn_vect[j] denotes the $\mathrm{P}_{\mathrm{j}}$ 's most recentminimum set) on the basis of cv vectors maintained at committed checkpoint's csn; on commit, for all j, $\mathrm{MSS}_{\mathrm{in}}$, which in case of Figure 4.2 is $\left\{\mathrm{P}_{1}, \mathrm{P}_{2}, \mathrm{P}_{4}\right\}$. (if int_vect $[j]==1$ ) comm_csn_vect[j]++; Therefore, $P_{2}$ sends ad hoc checkpoint request to $P_{1}$ and $P_{4}$ int_vect[] is the exact minimum set received alongand takes its own ad hoc checkpoint. After taking its ad with the commit request; comm_csn_vect[] is nothoc checkpoint, $\mathrm{P}_{1}$ sends $\mathrm{m}_{4}$ to $\mathrm{P}_{4} \cdot \mathrm{P}_{4} \operatorname{logs} \mathrm{m}_{4}^{-1}$ [Refer updated on tentative or ad hoc checkpoints; weSection 4.7 and 4.9]. In this case, $\mathrm{P}_{1}$ has taken its maintain one comm_csn_vect array for each MSScheckpoint before sending $\mathrm{m}_{4}$; at the time of receiving $\mathrm{m}_{4}$, and not for each process;

$\mathrm{P}_{4}$ has not taken its checkpoint for the current initiation. If

tnp_int_vect a bit vector of length $\mathrm{n}$; it contains the new $\mathrm{P}_{4}$ takes checkpoint after receiving $\mathrm{m}_{4}$, them $\mathrm{m}_{4}$ will processes found for the minimum set whilebecome orphan. Therefore $\mathrm{P}_{4} \operatorname{logs} \mathrm{m}_{4}^{-1}$. On recovery, $\mathrm{P}_{4}$ executing a potential checkpoint request [Referwill receive $\mathrm{m}_{4}$ as duplicate message because the processes Section 4.5.1]; are deterministic and $\mathrm{m}_{4}$ will be annihilated by $\mathrm{m}_{4}^{-1}$. Hence

np_int_vect a bit vector of length $\mathrm{n}$; it contains all newreceive of $\mathrm{m}_{4}$ as duplicate message will not cause any processes found for the minimum set at the MSS;nconsistency. It should be noted that this scheme is not on each potential checkpoint request: ifapplicable for non-deterministic systems. After taking its $($ tnp_int_vect $\neq \phi)$ np_int_vect= np_int_vect $\cup$ ad hoc checkpoint $C_{41}, P_{4}$ also finds that it was dependent tnp_int_vect $\quad$ upon $\mathrm{P}_{5}$ before taking the checkpoint due to $\mathrm{m}_{6}$ and $\mathrm{P}_{5}$ is

tint_vect a bit vector of length $n$; tint_vect $[\mathrm{k}]=1$ implies $\mathrm{P}_{\mathrm{k}}^{\text {not }}$ in the minimum set computed so far. Therefore, $\mathrm{P}_{4}$ belongs to the minimum set; it maintains the local sends ad hoc checkpoint request to $\mathrm{P}_{5}$. On receiving the knowledge of the minimum set; on receiving checkpoint request, $\mathrm{P}_{5}$ takes its ad hoc checkpoint. At time tint_vect, int_vect, tnp_int_vect along with ad_req ${ }^{t_{2}}, \mathrm{P}_{2}$ receives responses from all relevant processes and 
sends the tentative checkpoint request along with the minimum set $\left[\left\{\mathrm{P}_{1}, \mathrm{P}_{2}, \mathrm{P}_{4}, \mathrm{P}_{5}\right\}\right]$ to all processes. When a process, in the minimum set, receives the tentative checkpoint request, it converts its ad hoc checkpoint into tentative one. Finally, at time $t_{3}, P_{2}$ sends the commit message to all concerned processes. In this example, $\left\{\mathrm{C}_{00}\right.$, $\left.\mathrm{C}_{11}, \mathrm{C}_{21}, \mathrm{C}_{30}, \mathrm{C}_{41}, \mathrm{C}_{51}, \mathrm{~m}_{4}^{-1}\right\}$ constitute a recovery line. It should be noted that, in the recorded global state, $\mathrm{m}_{4}$ is an orphan message and its anti-message is also recorded at the receiver end.
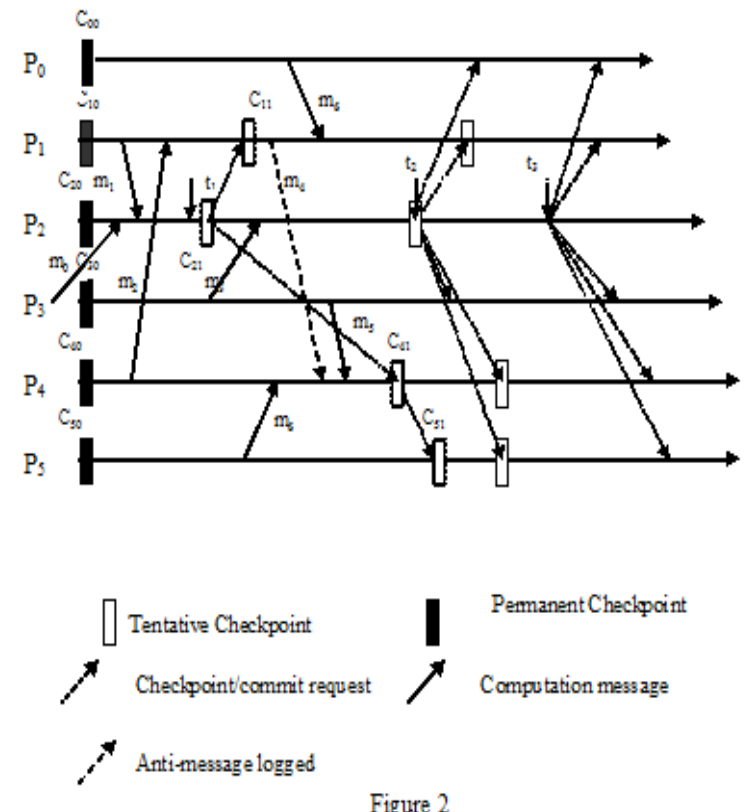

\subsubsection{The Proposed Checkpointing Algorithm}

When an MH sends an application message, it needs to first send to its local MSS over the wireless cell. The MSS can piggyback appropriate information onto the application message, and then route it to the appropriate destination. Conversely, when the MSS receives an application message to be forwarded to a local $\mathrm{MH}$, it first updates the relevant vectors that it maintains for the $\mathrm{MH}$, strips all piggybacked information from the message, and then forwards it to the MH. Thus, an MH sends and receives application messages that do not contain any additional information; it is only responsible for checkpointing its local state appropriately and transferring it to the MSS

Each process $\mathrm{P}_{\mathrm{i}}$ can initiate the checkpointing process. Initiator MSS (say $\mathrm{MSS}_{\text {in }}$ ) initiates and coordinates checkpointing process on behalf of $\mathrm{MH}_{\mathrm{i}}$. It computes int_vect (subset of the minimum set on the basis of direct dependencies maintained locally); and sends ad hoc checkpoint request (say ad-req) along with int_vect to an MSS if the later supports at least one process in the int_vect. It also updates its tint_vect on the basis of int_vect. We assume that concurrent invocations of the algorithm do not occur.
On receiving the ad-req, along with the int_vect from the initiator MSS, an MSS, say $\mathrm{MSS}_{\mathrm{i}}$, takes the following actions. It updates its tint_vect on the basis of int_vect. It sends the ad_req to $P_{i}$ if the following conditions are met: (i) $P_{i}$ is running in its cell (ii) $P_{i}$ is a member of the int_vect and (iii) ad_req has not been sent to $\mathrm{P}_{\mathrm{i}}$. If no such process is found, $\mathrm{MSS}_{\mathrm{i}}$ ignores the ad_req. Otherwise, on the basis of tint_vect, cv vectors of processes in its cell, initial cv vectors of other processes, it computes tnp_int_vect. If tnp_int_vect is not empty, $\mathrm{MSS}_{\mathrm{i}}$ sends ad_req along with tint_vect, tnp_int_vect to an MSS, if the later supports at least one process in the tnp_int_vect. $\mathrm{MSS}_{\mathrm{i}}$ updates np_int_vect, tint_vect on the basis of tnp_int_vect and initializes tnp_int_vect.

On receiving ad_req along with tint_vect, tnp_int_vect from some MSS, an MSS, say MSS $\mathrm{j}_{\mathrm{j}}$, takes the following actions. It updates its own tint_vect on the basis of received tint_vect, tnp_int_vect and finds any process $P_{k}$ such that $P_{k}$ is running in its cell, $P_{k}$ has not been sent ad_req and $P_{k}$ is in tnp_int_vect. If no such process exists, it simply ignores this request. Otherwise, it sends the ad hoc checkpoint request to $P_{k}$. On the basis of tint_vect, $\mathrm{cv}[]$ of its processes and initial cv[] of other processes, it computes tnp_int_vect. If tnp_int_vect is not empty, MSS $_{j}$ sends the checkpoint request along with tint_vect, tnp_int_vect to an MSS, which supports at least one process in the tnp_int_vect. $\mathrm{MSS}_{\mathrm{j}}$ updates np_int_vect, tint_vect on the basis of tnp_int_vect. It also initializes tnp_int_vect.

For a disconnected $\mathrm{MH}$, that is a member of minimum set, the MSS that has its disconnected checkpoint, converts its disconnected checkpoint into the required one.

When an MSS learns that all of its relevant processes have taken their ad hoc checkpoints successfully or at least one of its processes has failed to take its adhoc checkpoint, it sends the response message along with the np_int_vect to the initiator MSS. If, after sending the response message, an MSS receives the checkpoint request along with the tnp_int_vect, and learns that there is at least one process in tnp_int_vect running in its cell and it has not taken its tentative checkpoint, then the MSS requests such process to take checkpoint. It again sends the response message to the initiator MSS.

When the initiator MSS receives a response from some MSS, it updates its int_vect on the basis of np_int_vect, received along with the response. Finally, initiator MSS sends tentative checkpoint request to all the processes of the minimum set. In this case, if some process fails to take ad hoc checkpoint in the first phase, then concerned MHs need to abort their ad hoc checkpoints only. The effort of taking an ad hoc checkpoint is insignificant as compared to the tentative one. In this way, the loss of checkpointing effort, in case of an abort of the checkpointing procedure, is significantly low.

When a process in the minimum set receives the tentative checkpoint request, it converts its ad hoc checkpoint into tentative one. In the third phase, initiator MSS sends commit or abort to all processes. On receiving abort, a process discards its tentative checkpoint, if any, and undoes the updating of data structures. On receiving commit, processes, in the int_vect [], convert their tentative checkpoints into permanent ones. On receiving commit or abort, all processes update their dependency vectors and other data structures. 


\subsubsection{Handling Node Mobility and Disconnections}

Disconnection of an $\mathrm{MH}$ is a voluntary operation, and frequent disconnections of MHs is an expected feature of a mobile distributed system. Abrupt disconnections due to battery failure, process failure, or network failure are different from voluntary disconnections [1].

An MH may be disconnected from the network for an arbitrary period of time. The Checkpointing algorithm may generate a request for such $\mathrm{MH}$ to take a checkpoint. Delaying a response may significantly increase the completion time of the checkpointing algorithm. We propose the following solution to deal with disconnections that may lead to infinite wait state [1].

Suppose, an $\mathrm{MH}$, say $\mathrm{MH}_{\mathrm{i}}$, disconnects from the MSS, say $\mathrm{MSS}_{\mathrm{k}}$. MH $\mathrm{H}_{\mathrm{i}}$ takes its checkpoint, say d_ckpt $\mathrm{i}_{\mathrm{i}}$, and transfers it to $\mathrm{MSS}_{\mathrm{k}}$. MSS $\mathrm{K}_{\mathrm{k}}$ stores all the relevant data structures and $\mathrm{d}_{-} \mathrm{ckpt}_{\mathrm{i}}$ of $\mathrm{MH}_{\mathrm{i}}$ on stable storage. If $\mathrm{MH}_{\mathrm{i}}$ is in the int_vect[], d_ckpt is considered as $\mathrm{MH}_{\mathrm{i}}$ 's checkpoint for the current initiation. On commit, $\mathrm{MSS}_{\mathrm{k}}$ also updates $\mathrm{MH}_{\mathrm{i}}$ 's data structures, e.g., cv[], send, etc. On the receipt of messages for $M H_{i}, M S S_{k}$ does not update $M H_{i}$ 's $c v$ [], but maintains a message queue to store the messages.

When $\mathrm{MH}_{\mathrm{i}}$ enters in the cell of MSS , it is connected to the MSS $_{j}$ if no checkpointing process is going on. Before connection, $\mathrm{MSS}_{\mathrm{j}}$ collects its cv[], buffered messages, etc. from $\mathrm{MSS}_{\mathrm{k}}$; and $\mathrm{MSS}_{\mathrm{k}}$ discards $\mathrm{MH}_{\mathrm{i}}$ 's support information and d_ckpt $t_{\mathrm{i}}$. The stored messages are processed by $\mathrm{MH}_{\mathrm{i}}$, in the order of their receipt at the MSS. $\mathrm{MH}_{\mathrm{i}}$ 's cv[] is updated on the processing of buffered messages. If a node does not reconnect in a stipulated time, then its computation can be restarted from its d_ckpt.

\section{HANDLING FAILURES DURING CHECKPOINTING}

Since MHs are prone to failure, an MH may fail during checkpointing process. Sudden or abrupt disconnection of an $\mathrm{MH}$ is also termed as a fault [1]. Suppose, $\mathrm{P}_{\mathrm{i}}$ is waiting for a message from $P_{j}$ and $P_{j}$ has failed, then $P_{i}$ times out and detects the failure of $P_{j}$. If the failed process is not required to checkpoint in the current initiation or the failed process has already taken its tentative checkpoint, the checkpointing process can be completed uninterruptedly. If the failed process is not the initiator, one way to deal with the failure is to discard the whole checkpointing process similar to the approach in [4], [5]. The failed process will not be able to respond to the initiator's requests and initiator will detect the failure by timeout and will abort the current checkpointing process. If the initiator fails after sending commit or abort message, it has nothing to do for the current initiation. Suppose, the initiator fails before sending commit or abort message. Some process, waiting for the checkpoint/commit request, will timeout and will detect the failure of the initiator. It will send abort request to all processes discarding the current checkpointing process.

The above approach seems to be inefficient, because, the whole checkpointing process is discarded even when only one participating process fails. In our scheme, if any process fails to take its ad hoc checkpoint in the first phase, all concerned processes abort their ad hoc checkpoints only; and the loss of checkpointing effort is quite low as compared to other protocols $[2,4,3,6]$, in which every concerned process is forced to abort its tentative checkpoint. In our scheme, if any process fails to convert its ad hoc checkpoint into tentative one, then we propose to follow the technique proposed by Kim \& Park [7] in which a process commits its tentative checkpoints if none of the processes, on which it transitively depends, fails; and the consistent recovery line is advanced for those processes that committed their checkpoints. The initiator and other processes, which transitively depend on the failed process, have to abort their tentative checkpoints. Thus, in case of a node failure during second phase of checkpointing, total abort of the checkpointing is avoided.

\section{PERFORMANCE EVALUATION}

We use following notations to compare our algorithm with other algorithms:

$N_{m s s}: \quad$ number of MSSs.

$N_{m h}: \quad$ number of MHs.

$\mathrm{C}_{\mathrm{pp}}$ : $\quad$ cost of sending a message from one process to another

$C_{s t}: \quad$ cost of sending a message between any two MSSs.

$C_{w l}: \quad$ cost of sending a message from an $\mathrm{MH}$ to its local MSS (or vice versa).

$C_{b s t}$ : cost of broadcasting a message over static network.

$C_{\text {search }}$ : cost incurred to locate an $\mathrm{MH}$ and forward a message to its current local MSS, from a source MSS.

$T_{s t}: \quad$ average message delay in static network.

$T_{w l}$ : $\quad$ average message delay in the wireless network.

$T_{c h}$ : $\quad$ average delay to save a checkpoint on the stable storage. It also includes the time to transfer the checkpoint from an MH to its local MSS.

$N: \quad$ total number of processes

$N_{\text {min }}$ : number of minimum processes required to take checkpoints.

$N_{\text {mut }}$ : number of useless mutable checkpoints [2].

$N_{\text {ind }}$ : number of useless mutable checkpoints in the proposed protocol.

$T_{\text {search: }}$ average delay incurred to locate an $\mathrm{MH}$ and forward a message to its current local MSS.

$\mathrm{N}_{\text {ucr }}$ : average number of useless checkpoint requests in

[2].

$\mathrm{N}_{\text {dep }}$ : average number of processes on which a process depends.

The Synchronization message overhead:

In the first phase, a process taking an ad hoc checkpoint needs two system messages: request and reply. However, we have used some techniques to reduce the duplicate checkpoint requests. Thus the system overhead is approximately $2 * N_{\min } * C_{p p}$ in the first phase. Similarly, system overhead in the second phase is: $2 * \mathrm{~N}_{\min }{ }^{*} \mathrm{C}_{\mathrm{pp}}$. In the first phase we broadcast the adhoc checkpoint request. In the second phase, the tentative requested is broadcasted on the static network; and the system overhead is $\mathrm{C}_{\mathrm{bst}}$. In the third phase, we broadcast the commit request. The total message overhead comes out to be: $4 * \mathrm{~N}_{\text {min }} * \mathrm{C}_{\mathrm{pp}}+3 \mathrm{C}_{\mathrm{bst}}$ Number of processes taking checkpoints: It requires only minimum number of processes to take their checkpoints. In minimum-process coordinated checkpointing, some useless checkpoints are taken which are discarded on commit $[2,8,14]$; or some blocking of processes takes place during checkpointing $[4,6,9]$. In the proposed scheme, no useless checkpoints are taken and no blocking of processes takes place. We log anti-messages of very few 
messages at the receiver's end only during the checkpointing period. The effort of logging few antimessages may be negligibly small as compared to taking some useless checkpoints or blocking some processes during checkpointing especially in mobile distributed systems.

The blocking time of the Koo-Toueg [4] protocol is highest, followed by Cao-Singhal [6] algorithm. The other schemes are non-blocking [2,3,13], like the proposed one. In Elnozahy et al [3] algorithm, all processes are required to take their checkpoints in an initiation. In the protocols [6], [4], and the proposed one, only minimum numbers of processes record their checkpoints.

Table 1 A Comparison of System Performance

\begin{tabular}{|l|l|l|l|l|}
\hline & $\begin{array}{l}\text { Cao- } \\
\text { Singhal } \\
\text { [6] }\end{array}$ & $\begin{array}{l}\text { Koo- } \\
\text { Toeg } \\
\text { Algorith } \\
\text { m [4] }\end{array}$ & $\begin{array}{l}\text { Elnozah } \\
\mathbf{y} \\
\text { et al [3] }\end{array}$ & $\begin{array}{l}\text { Propos } \\
\text { ed } \\
\text { Algorit } \\
\text { hm }\end{array}$ \\
\hline $\begin{array}{l}\text { Avg. } \\
\text { blocking } \\
\text { Time }\end{array}$ & $2 T_{s t}$ & $\mathrm{~N}_{\text {min }} * \mathrm{~T}_{\text {ch }}$ & 0 & 0 \\
\hline $\begin{array}{l}\text { Average } \\
\text { No. of } \\
\text { checkpoints }\end{array}$ & $N_{\text {min }}$ & $N_{\text {min }}$ & $N$ & $N_{\text {min }}$ \\
\hline $\begin{array}{l}\text { Average } \\
\text { Message } \\
\text { Overhead }\end{array}$ & $\begin{array}{l}3 C_{b s t}+ \\
2 C_{\text {wireless }}+ \\
2 N_{m s s}^{*} C_{s t} \\
+3 N_{m h} *\end{array}$ & $\begin{array}{l}3 * \mathrm{~N}_{\text {min }} * \\
C_{\mathrm{pp}} * \mathrm{~N}_{\text {dep }}\end{array}$ & $\begin{array}{l}2 * C_{b s t}+ \\
C_{w l}\end{array}$ & $\begin{array}{l}4 * C_{\text {min }} * \\
C_{p p}+\end{array}$ \\
& & & $3 C_{b s t}$ \\
\hline
\end{tabular}

The message overhead in the proposed protocol is greater than $[2,3,4,6]$ due to the fact that the proposed scheme is a three phase algorithm. Our algorithm is a three phase algorithm; therefore it suffers from extra message overhead of $\mathrm{C}_{\mathrm{bst}}+2 \mathrm{~N}_{\min } * \mathrm{C}_{\mathrm{wl}}$. By doing so, we are able to reduce the loss of checkpointing effort in case of abort of the checkpointing procedure in the first phase. In other algorithms $[2,3,4,6,13]$, in case of abort in the first phase, all concerned processes are forced to abort their tentative checkpoint whereas in the proposed scheme, all relevant processes abort their ad hoc checkpoints only. The effort of taking an ad hoc checkpoint is negligible as compared to tentative one in the mobile distributed system [2]. Frequent abort of checkpointing algorithms, due to exhausted battery power, abrupt disconnections etc., may significantly increase the checkpointing overhead in twophase algorithms. We try to minimize the same by designing the three phase algorithm at the cost of slight increase in message overhead.

The algorithms proposed in $[2,3,4,6,8,9]$ assume that the processes are non-deterministic, whereas, we assume in the proposed algorithm that the processes are deterministic in nature as in [13].

\section{CONCLUSIONS}

In this chapter, we have proposed a minimum-process nonintrusive checkpointing protocol for deterministic mobile distributed systems, where no useless checkpoints are taken and no blocking of processes takes place. In minimum-process checkpointing protocols, some useless checkpoints are taken or blocking of processes takes place; we eliminate both by logging anti-messages of selective messages at the receiver end only during the checkpointing period. The overheads of logging a few anti-messages may be negligible as compared to taking some useless checkpoints or blocking the processes during checkpointing especially in mobile distributed system. We also try to reduce the loss of checkpointing effort when any process fails to take its checkpoint in coordination with others in the first phase. In case of a failure during checkpointing in the first phase, all concerned processes need to abort their ad hoc checkpoints only. The cost of taking an ad hoc checkpoint is negligibly small as compared to the tentative one especially in case of mobile distributed systems. In case, some process fails to convert its ad hoc checkpoint into tentative one, then we follow the selective commit mechanism, in which a process commits its checkpoint if none of the process, it causally depends upon, fails to take its tentative checkpoint. We disallow concurrent executions in spite of concurrent initiations of the proposed protocol.

\section{REFERENCES}

[1] Acharya A., "Structuring Distributed Algorithms and Services for networks with Mobile Hosts", Ph.D. Thesis, Rutgers University, 1995.

[2] Cao G. and Singhal M., "Mutable Checkpoints: A New Checkpointing Approach for Mobile Computing systems," IEEE Transaction On Parallel and Distributed Systems, vol. 12, no. 2, pp. 157-172, February 2001.

[3] Elnozahy E.N., Johnson D.B. and Zwaenepoel W., "The Performance of Consistent Checkpointing," Proceedings of the 11th Symposium on ReliableDistributed Systems, pp. 39-47, October 1992.

[4] Koo R. and Toueg S., "Checkpointing and Roll-Back Recovery for Distributed Systems," IEEE Trans. on Software Engineering, vol. 13, no. 1, pp. 23-31, January 1987.

[5] Prakash R. and Singhal M., "Low-Cost Checkpointing and Failure Recovery in Mobile Computing Systems," IEEE Transaction On Parallel and Distributed Systems, vol. 7, no. 10, pp. 10351048, October 1996.

[6]. G. Cao and M. Singhal. "On impossibility of MinProcess and Non-Blocking Checkpointing and An Efficient Checkpointing algorithm for mobile computing Systems". OSU Technical Report \#OSUCISRC-9/97-TR44, 1997.

[7] J.L. Kim, T. Park, " An efficient Protocol for checkpointing Recovery in Distributed Systems," IEEE Trans. Parallel and Distributed Systems, pp.955-960,Aug.1993.

[8] P. Kumar, L. Kumar and R.K. Chauhan, "A NonIntrusive minimum process synchronous checkpointing protocol for mobile distributed systems", in proceeding of IEEE ICPWC-2005,2005.

[9] Parveen Kumar, "A Low-Cost Hybrid Coordinated Checkpointing Protocol for mobile distributed systems", Mobile Information Systems. pp 13-32, Vol. 4, No. 1, 2007.

[10]David R. Jefferson, "Virtual Time", ACM Transactions on Programming Languages and Systems, Vol. 7, NO.3, pp 404-425, July 1985. 
[11] Johnson, D.B., Zwaenepoel, W., “ Sender-based message logging", In Proceedingss of $17^{\text {th }}$ international Symposium on Fault-Tolerant Computing, pp 14-19, 1987.

[12] Johnson, D.B., Zwaenepoel, W., "Recovery in Distributed Systems using optimistic message logging and checkpointing. pp 171-181, 1988.

[13] Pushpendra Singh, Gilbert Cabillic, "A Checkpointing Algorithm for Mobile Computing Environment", LNCS, No. 2775, pp 65-74, 2003.

[14]L. Kumar, M. Misra, R.C. Joshi, "Low overhead optimal checkpointing for mobile distributed systems" Proceedings. 19th IEEE International Conference on Data Engineering, pp 686 - 88, 2003.

[15] Parveen Kumar, Lalit Kumar, R K Chauhan, “A Nonintrusive Hybrid Synchronous Checkpointing Protocol for Mobile Systems", IETE Journal of Research, Vol. 52 No. 2\&3, 2006.

[16] Sunil Kumar, R K Chauhan, Parveen Kumar, "A Minimum-process Coordinated Checkpointing Protocol for Mobile Computing Systems", International Journal of Foundations of Computer science, Vol 19, No. 4, pp 1015-1038 (2008). 